DOI 10.15593/2224-9354/2017.2.17

УДК 336.14

Т.А. Найденова, И.Н. Швецова

\title{
МЕТОДИЧЕСКИЙ ИНСТРУМЕНТАРИЙ ОЦЕНКИ ФИНАНСОВОЙ УСТОЙЧИВОСТИ БЮДЖЕТОВ СУБЪЕКТОВ РОССИЙСКОЙ ФЕДЕРАЦИИ
}

\begin{abstract}
Современной парадигмой территориального развития, в соответствии с которой территории должны стремиться к экономическому росту за счет собственных ресурсов, выступает саморазвитие социально-экономических систем. При этом одним из важнейших факторов саморазвития экономики территории выступает достаточность бюджетных ресурсов для выполнения органами государственной власти публично-правовых образований закрепленных за ними функций. В этой связи большое научно-практическое значение приобретает совершенствование методического инструментария комплексной оценки финансовой устойчивости бюджетов субъектов РФ, что определяет предмет данной научной статьи.

Представлен методический инструментарий комплексной оценки финансовой устойчивости бюджетов субъектов Российской Федерации.

Предложенный инструментарий комплексной оценки фринансовой устойчивости бюджетов субъектов Российской Федерации может быть использован органами государственной власти публично-правовых образований при принятии управленческих финансовых решений; необходим инвесторам (отечественным и зарубежным) для проведения независимых оценок финансовой ситуации в конкретном субъекте Российской Федерации при обосновании инвестиционных решений.

Методика комплексной оценки финансовой устойчивости субъектов Российской Федерации отвечает критериям объективности, конкретности полученных результатов. Использование данной методики позволит провести комплексный анализ устойчивости бюджета, сделать обоснованные выводы о финансовом состоянии территории, принять взвешенные решения при вложении бюджетных средств руководителям субъектов Российской Федерации, инвесторам реально оценить риск вложения капитала в те или иные публично-правовые образования.

Ключевые слова: бюджет, устойчивость бюджета, методика комплексной оценки, инструментарий оценки.
\end{abstract}

Введение. Необходимость совершенствования имеющегося методического инструментария оценки финансовой устойчивости региональных бюджетов может быть обоснована совокупностью взаимосвязанных обстоятельств.

Первое обстоятельство обусловлено тем, что в новых макроэкономических условиях в «Программе повышения эффективности управления общественными (государственными и муниципальными) финансами на период до 2018 года» [1], в Государственной программе «Управление государственными финансами и регулирование финансовых рынков» [2], а также в Бюджетном послании Президента РФ Федеральному собранию «О бюджетной поли-

(с) Найденова Т.А., Швецова И.Н., 2017

Найденова Татьяна Анатольевна - канд. экон. наук, доцент кафедры банковского дела ФГБОУ ВО «Сыктывкарский государственный университет им. Питирима Сорокина», e-mail: NaydenovaTA@mail.ru.

Швецова Ирина Николаевна - канд. экон. наук, доцент, заведующая кафедрой финансового менеджмента ФГБОУ ВО «Сыктывкарский государственный университет им. Питирима Сорокина», e-mail: irshv@mail.ru. 
тике в 2014-2016 годах» [3] в качестве первоочередных определена стратегическая цель обеспечения долгосрочной устойчивости бюджетов субъектов Российской Федерации как базового принципа ответственной бюджетной политики. В связи с этим в долгосрочной перспективе повышаются требования к методическому инструментарию оценки финансовой устойчивости бюджета субъекта Российской Федерации.

Второе обстоятельство детерминировано тем, что в условиях конкуренции и возрастания влияния внешних факторов на процесс регионального воспроизводства проблемы саморазвития территориальных социально-экономических систем приобретают особую значимость [4, с. 218]. Саморазвитие - это способность региона в условиях сложившейся в обществе макросреды обеспечить расширенное воспроизводство валового регионального продукта за счет собственных доходных источников [5, с. 60]. С позиции системного подхода саморазвитие территорий возможно только при наличии бюджетных ресурсов в объемах, необходимых органам государственной власти публично-правовых образований для полного выполнения закрепленных за ними функций, достаточность которых определяется степенью устойчивости бюджетов, что требует комплексной оценки финансовой устойчивости регионального бюджета.

Третье обстоятельство связано с тем, что в экономической теории устойчивость рассматривается в логической взаимосвязи с понятиями равновесия $[6$, с. 131$]$, сбалансированности [7, с. $715-717 ; 8$, с. 259 ; 9, с. 270]. Самостоятельность и платежеспособность публично-правового образования, как правило, остаются за рамками исследования. Поэтому возникает необходимость разработки методики комплексной оценки финансовой устойчивости бюджета публично-правового образования.

Четвертое обстоятельство вытекает из требований Бюджетного кодекса РФ к состоянию региональных и местных бюджетов. Требования включают в себя ограничения по уровню государственного долга, по объему расходов на его обслуживание, по уровню дефицита бюджета, по соблюдению установленных нормативов расходов на содержание органов государственной власти условиям предоставления межбюджетных трансфертов и др. [10]. Это позволяет сделать вывод, что их выполнение трактуется как основа обеспечения бюджетной устойчивости, а следовательно, возникает необходимость совершенствования методики комплексной оценки финансовой устойчивости бюджета субъекта Российской Федерации.

В силу представленных аргументов совершенствование методического инструментария комплексной оценки финансовой устойчивости бюджетов субъектов РФ является актуальной задачей.

Методология оценки устойчивости бюджетов субъектов Российской Федерации. Методология оценки устойчивости региональных бюджетов - это инструмент оценки состояния региональных бюджетов, имеющихся тенденций, идентификации проблемных моментов. 
Методология оценки финансовой устойчивости бюджетов публичноправовых образований включает в себя два элемента: концепцию оценки и набор методик оценки устойчивости бюджетов субъектов Российской Федерации.

Конщепщия оценки устойчивости бюджетов. Существуют различные подходы к определению параметров бюджетной устойчивости. Чаще всего устойчивость оценивают по критерию «сбалансированность бюджета» [11, с. 15].

Г.Б. Поляк устойчивость бюджета оценивает через показатели самостоятельности бюджета [12, с. 682]. Параметры доходов, расходов и их соотношение сравниваются со средними значениями по стране, а также с зафиксированными по данной группе регионов. Проводятся межвременные сравнения значений индикаторов за ряд лет. Эти подходы применяются для оценки преимущественно результирующих параметров развития системы. Возможно сравнение значений индикаторов с неким идеальным состоянием, конструируемым искусственно [13, с. 13-14].

В любом случае оценка состоит в сравнении определенного набора индикаторов с их целевыми значениями и формулировании выводов. При этом выделяются интервалы соответствия значений индикаторов нормальному состоянию или целевым характеристикам [14, с. 14].

Различают количественные и качественные индикаторы. Количественные индикаторы характеризуют результаты развития объекта, и главная задача здесь - установить взаимное соответствие значения индикаторов и наличия проблем. Качественные индикаторы относятся в большей степени к характеристикам системы управления и отражают степень соответствия элементов системы управления целевому значению.

На наш взгляд, при оценке устойчивости отсутствует комплексный подход, что не позволяет дать объективную картину состояния бюджета, при котором возможно стабильное функционирование публично-правового образования с учетом предусмотренных бюджетом расходных обязательств.

Методики оценки устойчивости бюджета. В настоящее время проблема отсутствия прозрачных унифицированных методик определения устойчивости бюджета в целях повышения эффективности функционирования бюджетной системы, а также поддержки комплексного социально-экономического развития территории остро встает перед органами государственной власти, инвесторами и кредиторами. Это делает чрезвычайно актуальной необходимость изучения и разработки новых методологических подходов к проведению комплексной оценки бюджетной устойчивости субъектов РФ на основе специально разработанной системы показателей оценки бюджетной устойчивости.

Для анализа финансовой устойчивости бюджетов субъектов РФ в качестве показателей предлагается использовать относительные показатели состояния бюджета, рассчитываемые как отношения абсолютных бюджетных показателей друг к другу.

Сравнительный анализ методик оценки устойчивости регионального бюджета представлен в табл. 1. 


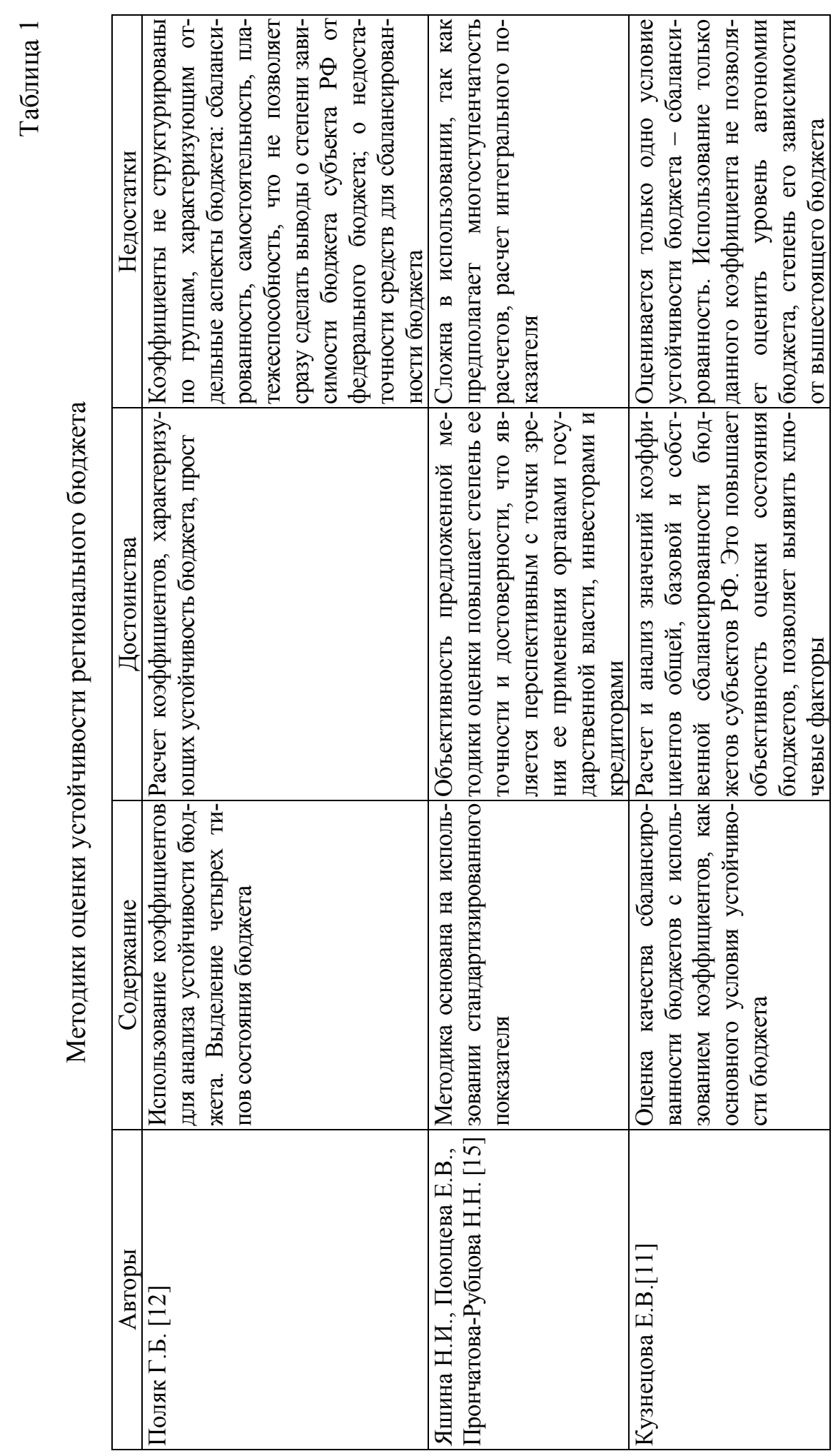

PNRPU Sociology and Economics Bulletin. 2017. No. 2 
Рассматривая и оценивая современные методики оценки устойчивости бюджета, отметим особую значимость унифицированной методики, предложенной профессором Г.Б. Поляком. Степень финансовой устойчивости имеет четыре уровня: абсолютно устойчивое состояние, нормальное состояние, неустойчивое состояние, кризисное состояние.

В качестве оценочных показателей предположены: расходы бюджета, доходы бюджета, бюджетная задолженность (табл. 2).

Таблица 2

Степень финансовой устойчивости бюджета

\begin{tabular}{|c|c|c|}
\hline Тип устойчивости & Условие & Примечание \\
\hline Абсолютно устойчивое состояние & $\mathrm{P}_{\mathrm{мин}}=Д_{\mathrm{c}}+Д_{\Pi}$ & \multirow{4}{*}{$\begin{array}{l}\mathrm{P}_{\text {мин }} \text {-расходы минимальные; } \\
\text { Д }_{\text {c доходы собственные; }} \\
\text { Д }_{\text {п доходы привлеченные; }} \\
\text { Д }_{\text {д дополнительно при- }} \text { влеченные финансовые ре- } \\
\text { сурсы (свободные остатки } \\
\text { бюджетных средств, сред- } \\
\text { ства целевых бюджетных } \\
\text { фондов и др.) }\end{array}$} \\
\hline Нормальное состояние & $\mathrm{P}_{\mathrm{мин}}=Д_{\mathrm{c}}+Д_{\Pi}$ & \\
\hline Неустойчивое состояние & $P_{\mathrm{мин}}=Д_{c}+Д_{\Pi}+Д_{д}$ & \\
\hline Кризисное состояние & $\mathrm{P}_{\text {мин }}=Д_{\mathrm{c}}+Д_{п}$ & \\
\hline
\end{tabular}

Источник: [12, с. 681].

Абсолютно устойчивое состояние характеризуется достаточной величиной собственных доходов для обеспечения всех расходных полномочий субъекта РФ, сбалансированностью бюджета, приоритетностью финансирования социальных расходов.

Нормальное состояние отражает достаточную величину доходов для покрытия минимальных расходов, сбалансированность бюджета.

Неустойчивое положение бюджета возникает в случае, когда минимальные расходы могут быть покрыты только благодаря привлечению дополнительных ресурсов.

Кризисное состояние наступает в случае, когда собственных и привлеченных доходов недостаточно для покрытия минимальных расходов.

Количественные критерии для определения степени устойчивости бюджета представлены в табл. 3.

Исходя из экономического и налогового потенциала территорий приведенные соотношения собственных и перераспределяемых доходов могут быть различными для различных регионов, поэтому эти соотношения должны быть определены на основе анализа для каждого субъекта Российской Федерации.

По мнению Г.Б. Поляка, в анализе бюджета в качестве инструментария могут использоваться бюджетные коэффициенты, в частности, коэффициент соотношения перераспределяемых и собственных доходов, коэффициент автономии, коэффициент внешнего финансирования, коэффициент обеспечен- 
ности минимальных расходов собственными доходами, коэффициент бюджетного покрытия, коэффициент налогового покрытия, коэффициент неналогового покрытия, коэффициент трансфертного покрытия, коэффициент дефицитности бюджета, коэффициент бюджетной задолженности, коэффициент бюджетной результативности региона, коэффициент бюджетной обеспеченности населения [12, с. 682].

Таблица 3

Количественные критерии определения устойчивости бюджета

\begin{tabular}{|c|c|c|}
\hline Тип устойчивости & Условие & Примечание \\
\hline Абсолютно устойчивое состояние & $\begin{array}{c}Д_{c} / \text { Д }=60-70 \% ; \\
Д_{\Pi} / \text { Д }=30-40 \% ; \\
3 / P=10-15 \%\end{array}$ & \multirow{3}{*}{$\begin{array}{l}\text { Д }- \text { доходы собственные; } \\
\text { Д - доходы бюджета; } \\
\text { P - расходы бюджета; } \\
3 \text { - бюджетная задолженность }\end{array}$} \\
\hline Нормальное состояние & $\begin{array}{c}Д_{c} / \text { Д }=40-50 \% ; \\
Д_{\Pi} / \text { Д }=50-60 \% ; \\
3 / P=30-35 \%\end{array}$ & \\
\hline Неустойчивое состояние & $\begin{array}{c}Д_{c} / \text { Д }=5-10 \% ; \\
Д_{\Pi} / \text { Д }=90-95 \% ; \\
3 / P=40-50 \%\end{array}$ & \\
\hline
\end{tabular}

Источник: [12, с. 681].

С помощью названных инструментов достигается объективизация в оценивании состояния территориальных бюджетов, выявляются факторы, влияющие на это состояние. Они могут применяться для сравнения показателей бюджета за различные периоды времени, для сравнения показателей бюджета конкретной территории с аналогичными показателями бюджетов других территорий. Эти коэффициенты могут быть использованы в качестве нормативов (критериев) состояния бюджета, которые могут разрабатываться на базе наиболее успешно составленных и исполненных бюджетов, на базе групп бюджетов, сформированных по территориальному признаку и др.

Исследование устойчивости бюджетов по системе критериев неоднозначно характеризует эффективность управления ими из-за проблемы сопоставления и выделения наиболее и наименее устойчивых бюджетов. В связи с этим Н.И. Яшина, Е.В. Поющева, Н.Н. Прончатова-Рубцова предлагают рассчитать сводный стандартизированный показатель для конкретного бюджета [15]. Суть методики заключается в том, что в процессе экономической оценки хозяйствующих субъектов рассчитанные показатели системы бюджетной устойчивости могут иметь разную размерность, важность или весомость. Требование комплексности в оценке бюджетов регионов по совокупности показателей делает необходимым приведение их к безразмерному виду, к общему началу отсчета, к единому интервалу изме- 
нения. Этого можно добиться, прибегнув к методу, базирующемуся на преобразовании исходных показателей [15].

Формируются следующие классы:

1) «лучшие бюджеты» - обладают наименьшими из выборки значениями сводного стандартизированного показателя, не превышающими значения порогового показателя для отнесения к этому классу;

2) «удовлетворительные бюджеты» (средний класс) - значения сводного стандартизированного показателя больше значений I класса, но меньше пороговых значений II класса;

3) «худшие бюджеты» - значения с наибольшими значениями сводного стандартизированного показателя, худшие из выборки.

Е.В. Кузнецова выделяет следующие виды сбалансированности [11, с. 16]:

1) общая сбалансированность бюджета, которая учитывает возвратные финансовые ресурсы;

2) базовая сбалансированность, которая учитывает суммы доходов бюджета субъекта РФ, включая межбюджетные трансферты;

3) собственная сбалансированность, которая не учитывает безвозмездных поступлений.

Для количественной оценки качества сбалансированности бюджетов рассчитываются коэффициенты, характеризующие соотношение источников финансового обеспечения расходов и общей суммы расходов региональных бюджетов, в разрезе выделенных видов сбалансированности. Предлагаемые коэффициенты могут также использоваться для сравнения показателей финансового состояния отдельных субъектов РФ с аналогичными показателями других публично-правовых образований, выявления динамики их развития и тенденций изменения финансового состояния.

Проведя анализ подходов к оценке устойчивости бюджетов, оценив их достоинства и недостатки, предлагаем методику комплексной оценки финансовой устойчивости бюджетов. В предлагаемой методике учитываются следующие принципы: доступность, простота и однозначность заданных показателей; использование точных статистических данных об исполнении бюджета; оценка первичных показателей во времени и в относительном выражении.

Поскольку критериями устойчивости бюджета выступают сбалансированность, самостоятельность и платежеспособность, которые характеризуют способность публично-правовых образований мобилизовать собственные финансовые ресурсы в бюджет, а также полно и своевременно отвечать по своим обязательствам, то для получения более объективной оценки используем три группы индикаторов: 1) показатели, характеризующие сбалансированность бюджета; 2) показатели, характеризующие самостоятельность бюджета; 3) показатели, характеризующие платежеспособность. 
Первая группа индикаторов (характеризует сбалансированность бюджета) включает в себя:

1. Коэффициент общей сбалансированности бюджета. Он отражает соотношение расходов бюджета и источников финансирования. Определяется как отношение доходов бюджета и источников финансирования дефицита бюджета к расходам бюджета.

2. Коэффициент базовой сбалансированности. Показывает степень покрытия расходов бюджета за счет доходов, при этом из расчета исключаются источники финансирования дефицита бюджета, что позволяет оценить бюджетную сбалансированность, достигаемую без привлечения заемных инструментов. Определяется как отношение доходов бюджета к расходам бюджета.

3. Коэффициент собственной сбалансированности. Показывает степень покрытия расходов бюджета на реализацию собственных полномочий публично-правового образования за счет доходов без учета безвозмездных поступлений, объем которых не зависит от деятельности органов власти публично-правового образования. Определяется отношением общей суммы доходов бюджета за вычетом безвозмездных поступлений к расходам бюджета за вычетом субвенций.

Вторая группа индикаторов (характеризует самостоятельность бюджета) включает в себя:

1. Коэффициент бюджетной независимости. Показывает уровень автономии бюджета. Определяется отношением доходов собственных к общей сумме доходов бюджета.

2. Коэффищиент бюджетной зависимости. Отражает уровень зависимости бюджета от вышестоящего бюджета. Определяется отношением привлеченных доходов к общей сумме доходов бюджета.

3. Коэффициент обеспеченности минимальных расходов собственными доходами. Отражает уровень обеспеченности минимальных расходов собственными доходами. Определяется как отношение собственных доходов к расходам минимальным.

4. Коэффиџиент бюджетного покрытия. Показывает, насколько доходы бюджета покрывают минимальные расходы. Определяется как отношение доходов бюджета к минимальным расходам.

5. Коэффициент налогового покрытия. Показывает уровень покрытия минимальных расходов налоговыми доходами. Определяется как отношение налоговых доходов к минимальным расходам.

6. Коэффициент неналогового покрытия. Показывает уровень покрытия расходов неналоговыми доходами. Определяется как отношение неналоговых доходов к минимальным расходам.

7. Коэффициент трансфертного покрытия. Отражает степень покрытия расходов трансфертами из федерального бюджета. Определяется как от- 
ношение доходов бюджета, поступивших в виде трансфертов из федерального бюджета к расходам.

8. Коэффициент дефицитности бюджета. Показывает уровень дефицита бюджета. Определяется как отношение дефицита бюджета к расходам.

9. Коэффициент бюджетной задолженности. Показывает уровень бюджетной задолженности. Определяется как отношение задолженности бюджета к расходам.

Третья группа индикаторов (характеризует платежеспособность) включает в себя:

1. Коэффищиент общей платежеспособности. Характеризует способность субъекта покрывать расходы имеющимися в его распоряжении доходами. Определяется как отношение общей суммы доходов к расходам.

2. Коэффициент абсолютной платежеспособности. Характеризует способность субъекта покрывать социально значимые расходы имеющимися в его распоряжении доходами. Определяется как отношение общей суммы доходов бюджета к социально значимым расходам.

3. Коэффициент собственной платежеспособности. Характеризует достаточность собственных бюджетных доходов для финансирования расходных обязательств. Определяется как отношение суммы налоговых и неналоговых доходов к расходам.

Результирующими показателями устойчивости бюджета являются бюджетная результативность региона и бюджетная обеспеченность региона.

1. Коэффициент бюджетной результативности региона. Показывает сумму бюджетных доходов, созданных на территории, приходящихся на одного жителя. Определяется как отношение доходов бюджета на численность населения.

2. Коэффициент бюджетной обеспеченности региона. Отражает сумму общественных товаров и услуг, которую фактически получает один житель региона. Определяется как отношение общей суммы расходов на численность населения.

Количественная оценка данных элементов составит эмпирическую базу для мониторинга и анализа устойчивости бюджета, цель которых - выявление факторов, влияющих на уровень сбалансированности, самостоятельности и платежеспособности бюджета.

Заключение. Методика комплексной оценки финансовой устойчивости субъектов РФ свидетельствует об объективности и конкретности полученных результатов. Применение предложенного инструментария позволит органам власти публично-правовых образований своевременно выявлять риски вложения капитала в те или иные публично-правовые образования и адекватно реагировать на вызовы времени, сохранять платежеспособность в долгосрочной перспективе. 


\section{Список литературы}

1. Программа повышения эффективности управления общественными (государственными и муниципальными) финансами на период до 2018 года [Электронный ресурс]: утв. распоряжением Правительства Рос. Федерации от 30 дек. 2013 г. № 2593-p. - URL: www.consultant.ru (дата обращения: 12.10.2016).

2. Управление государственными финансами и регулирование финансовых рынков: гос. программа Рос. Федерации [Электронный ресурс]: утв. Постановлением Правительства Рос. Федерации от 15 апр. 2014 г. № 320. URL: www.consultant.ru (дата обращения: 12.10.2016).

3. О бюджетной политике в 2014-2016 годах [Электронный ресурс]: Бюджетное послание Президента РФ Федер. собранию от 13.06.2013. - URL: www.consultant.ru (дата обращения: 12.10.2016).

4. Пространственное развитие экономики макрорегиона (на примере Северо-Западного федерального округа): моногр. / под ред. С.В. Кузнецова; С.-Петерб. гос. ун-т аэрокосм. приборостроения. - СПб., 2013. - 334 с.

5. Татаркин А.И., Татаркин Д.А. Саморазвитие регионов в контексте федеративных отношений // Пространственная экономика. - 2008. - № 4. - С. 60-70.

6. Ротбард М. Власть и рынок: Государство и экономика / пер. с англ. Б.С. Пинскера. - Челябинск: Социум, 2003. - 415 с.

7. Абель Э., Бернанке Б. Макроэкономика. - 5-е изд. - СПб.: Питер, 2012. $-768 \mathrm{c}$.

8. Макконел К.Р., Брю С.Л. Экономикс: Принципы, проблемы и политика: в 2 т: пер. с англ. - Т. 1. - М.: Республика, 1992. - 399 с.

9. Мэнкью Н., Тейлор М. Микроэкономика. - 2-е изд. - СПб.: Питер, 2015. $-544 \mathrm{c}$.

10. Бюджетный кодекс РФ [Электронный ресурс]: Федер. закон от 31 июля 1998 г. № 145-Ф3 (ред. от 26.12.2014, с изм. от 08.03.2015). - URL: http://www.consultant.ru (дата обращения: 12.10.2016).

11. Кузнецова Е.В. Оценка качества сбалансированности бюджетов субъектов Российской Федерации // Финансы. - 2011. - № 2. - С. 15-19.

12. Бюджетная система России / под ред. Г.Д. Поляка. - 2-е изд., перераб. и доп. - М.: ЮНИТИ-ДАНА, 2008. - 703 с.

13. Клисторин В.И. Диагностика региональных финансов // Регион: экономика и социология. - 2004. - № 2. - С. 3-22.

14. Казаковцева М.В. Методологическое обеспечение влияния факторов на финансовую устойчивость бюджетов субъектов РФ // Вектор науки ТГУ. 2012. - № 3 (21). - С. 152-155.

15. Яшина Н.И., Поющева Е.В., Прончатова-Рубцова Н.Н. Совершенствование методологии определения бюджетной устойчивости субъектов РФ: два подхода к оценке в современных социально-экономических условиях [Электронный ресурс] // Бухгалтерский учет в бюджетных и некоммерческих организациях. - 2013. - № 9. - URL: www.consultant.ru (дата обращения: 12.10.2016). 


\section{References}

1. Programma povysheniia effektivnosti upravleniia obshchestvennymi (gosudarstvennymi i munitsipal'nymi) finansami na period do 2018 goda: utv. rasporiazheniem Pravitel'stva Ros. Federatsii ot 30 dek. 2013 g. № 2593-r [Program of public (state and municipal) finance management improvement for the period until 2018: approved by the Order of the Government of the Russian Federation of 30 December 2013 no. 2593-r]. Available at: www.consultant.ru (accessed 12 October 2016).

2. Upravlenie gosudarstvennymi finansami i regulirovanie finansovykh rynkov: gos. programma Ros. Federatsii: utv. Postanovleniem Pravitel'stva Ros. Federatsii ot 15 apr. 2014 g. № 320 [Public finances management and financial markets regulation: State. Program of Russian Federation: approved by Government Decree of Russian Federation of 15 April, 2014 no. 320]. Available at: www.consultant.ru (accessed 12 October 2016).

3. O biudzhetnoi politike v 2014-2016 godakh: Biudzhetnoe poslanie Prezidenta RF Feder. sobraniiu ot 13.06.2013 [On budgetary policy in 2014-2016: Budget message of the President of the Russian Federation to Federal Assembly of 13 June 2013]. Available at: www.consultant.ru (accessed 12 October 2016).

4. Prostranstvennoe razvitie ekonomiki makroregiona (na primere SeveroZapadnogo federal'nogo okruga) [Spatial development of the macro regional economy (the case study of the North-Western Federal District)]. Ed. by S.V. Kuznetsov, S.-Peterb. gos. un-t aerokosm. priborostroeniia. Saint-Petersburg, 2013, 334 p.

5. Tatarkin A.I., Tatarkin D.A. Samorazvitie regionov v kontekste federativnykh otnoshenii [Regional self-development in the context of federative relations]. Prostranstvennaia ekonomika, 2008, no. 4, pp. 60-70.

6. Rothbard M.N. Power and Market: Government and the economy. Institute for Human Studies Publ., 1970 [Russ. ed.: Rotbard M. Vlast' i rynok: Gosudarstvo i ekonomika. Cheliabinsk, Sotsium Publ., 2003, 415 p.].

7. Abel' E., Bernanke B. Makroekonomika [Macroeconomy]. 5th ed. SaintPetersburg, Piter Publ., 2012, 768 p.

8. McConnell C.R., Brue S.L. Economics: Principles, problems and policies. McGraw Hill, 1990 [Russ. ed.: Makkonel K.R., Briu S.L. Ekonomiks: Printsipy, problemy i politika. In 2 vol., vol. 1. Moscow, Respublika Publ., 1992, 399 p.].

9. Menk'iu N., Teilor M. Mikroekonomika [Microeconomy]. 2nd ed. SaintPetersburg, Piter Publ., 2015, 544 p.

10. Biudzhetnyi kodeks RF: Feder. zakon ot 31 iiulia 1998 g. № 145-FZ (red. ot 26.12.2014, s izm. ot 08.03.2015) [Budgetary Code of the Russian Federation: Federal Law of 31 July 1998 No. 145-FZ (as amended on 26 December 2014, as amended on 8 March 2015)]. Available at: http://www.consultant.ru (accessed 12 October 2016).

11. Kuznetsova E.V. Otsenka kachestva sbalansirovannosti biudzhetov sub"ektov Rossiiskoi Federatsii [Assessment of the budget balance of the constituent entities of the Russian Federation]. Finansy, 2011, no. 2, pp. 15-19. 
12. Biudzhetnaia sistema Rossii [Budgetary system of Russia]. Ed. by G.D. Poliak, 2nd ed., revised and corrected. Moscow, IuNITI-DANA Publ., 2008, 703 p.

13. Klistorin V.I. Diagnostika regional'nykh finansov [Regional finance diagnostics]. Region: ekonomika i sotsiologiia, 2004, no. 2, pp. 3-22.

14. Kazakovtseva M.V. Metodologicheskoe obespechenie vliianiia faktorov na finansovuiu ustoichivost' biudzhetov sub"ektov RF [Methodological influence of the factors on the budget's financial stability in the constituent entities of the Russian Federation]. Vektor nauki TGU, 2012, no. 3, vol. 21, pp. 152-155.

15. Iashina N.I., Poiushcheva E.V., Pronchatova-Rubtsova N.N. Sovershenstvovanie metodologii opredeleniia biudzhetnoi ustoichivosti sub"ektov RF: dva podkhoda $\mathrm{k}$ otsenke v sovremennykh sotsial'no-ekonomicheskikh usloviiakh [Methodological development for determining the budget sustainability of the constituent entities of the Russian Federation: two approaches to the assessment under contemporary socio-economic conditions]. Bukhgalterskii uchet $\mathrm{v}$ biudzhetnykh i nekommercheskikh organizatsiiakh, 2013, no. 9. Available at: www.consultant.ru (accessed 12 October 2016).

Оригинальность статьи - $75 \%$

Получено 18.01.2017

\section{T.A. Naydyonova, I.N. Shvetsova}

\section{METHODICAL TOOLKIT FOR THE ASSESSMENT OF THE RUSSIAN FEDERATION CONSTITUENT TERRITORIES' FINANCIAL STABILITY}

Self-development of socio-economic systems represents a modern paradigm of territorial development. It tells that the territories should develop themselves by their own resources. One of the key factors of territorial economic self-development is the sufficiency of budgetary resources, so that public authorities could fulfill their functions. Thus, the development of methodical toolkit for comprehensive financial stability assessment on the Russian Federation constituent territories is of practical and scientific importance. The purpose of the article is to develop a methodical toolkit for comprehensive assessment of the RF constituent territories' financial stability. The research findings include the toolkit and the recommendations for it to be applied by the public authorities in management and financial decision making. This is necessary for both Russian and foreign investors in order to carry out the independent assessment of financial environment on a particular territory of the RF to make a reasonable investment decision. The methodology of comprehensive assessment applied in the research meets the objectiveness criteria and the accuracy of the obtained results. Using this methodology allows to perform a comprehensive analysis of financial stability in a certain governmental unit. It also helps public authorities to make valid conclusions about the risks of budgetary funds investment in certain entities. Keywords: budget, financial stability, assessment methodology.

Tatyana A. Naydyonova - Candidate of Economic Sciences, Associate Professor, Dept. of Banking, Syktyvkar State University named after Pitirim Sorokin, e-mail: NaydenovaTA@mail.ru.

Irina N. Shvetsova - Candidate of Economic Sciences, Associate Professor, Dept. of Financial Management, Syktyvkar State University named after Pitirim Sorokin, e-mail: irshv@mail.ru. 